\title{
RENDIMIENTO POBLACIONAL DEL ROTÍFERO NATIVO Brachionus sp. “Cayman”, UTILIZANDO DIFERENTES ENRIQUECEDORES
}

\section{GROWTH PERFORMANCE OF THE NATIVE ROTIFER Brachionus sp. "Cayman" USING DIFFERENT ENRICHMENT SUPLEMENTS}

\author{
Rosario Cisneros ${ }^{1}$
}

\begin{abstract}
Resumen
Los rotíferos Brachionus spp. son cultivados y utilizados como alimento vivo para larvas de peces y crustáceos en todo el mundo. Con el objetivo de evaluar el rendimiento poblacional del rotífero nativo Brachionus sp. "Cayman", cepa Chilca, Perú, se realizó una prueba de enriquecimiento largo, utilizando cuatro enriquecedores/suplementos alimenticios comerciales, Marol-E, Protein Hufa, Selcon e Easy Super Selco, así como la microalga Nannochloropsis oculata sin suplemento, como control. Cada tratamiento se realizó por triplicado en tanques de 180 litros. Se determinó la fecundidad $(F)$, tasa de crecimiento poblacional $(T C)$ y productividad $(R)$ obtenida con cada tratamiento. El ANOVA mostró diferencias estadísticas entre los tratamientos, el enriquecedor Protein Hufa presento mejores resultados en cuanto a $F(0.65 \pm 0.03$ huevos.indiv $\left.{ }^{1}\right)$, densidad final $\left(149 \pm 0.58\right.$ indiv. $\left.\mathrm{mL}^{-1}\right), T C\left(0.38 \pm 0.02\right.$ día $\left.{ }^{-1}\right)$ y $R\left(29250 \pm 0.66\right.$ rot. $\mathrm{L}^{-1}$.día $\left.{ }^{-1}\right)$.
\end{abstract}

Palabras clave: cultivo, rotíferos, enriquecimiento, fecundidad, rendimiento.

\begin{abstract}
The rotifer Brachionus spp. is the most important live food for fish and crustacean cultures throughout the world. In order to improve the growth performance of native Brachionus sp. "Cayman," Chilca strain, Perú, we performed an experimental study of long-term enrichment with four commercial supplements, Marol-E, Protein Hufa, Selcon, Easy Super Selco and the microalgae Nannochloropsis oculata as control. The experiment was carried out in 180 liter tanks with three replicates per treatment. We determined the egg ratio $(F)$, population growth rate $(T C)$ and productivity $(R)$. ANOVA showed significant differences among treatments, Protein Hufa supplementation showed better results in terms of egg ratio $\left(0.65 \pm 0.03\right.$ eggs.ind $\left.^{-1}\right)$, final density $\left(149 \pm 0.58\right.$ indiv. $\left.\mathrm{mL}^{-1}\right)$, population growth rate $\left(0.38 \pm 0.02\right.$ día $\left.^{-1}\right)$ and productivity $(29250 \pm 0.66$ rot. $L^{-1}$. day $\left.^{-1}\right)$.
\end{abstract}

Key words: culture, rotifers, enrichment, egg ratio, growth performance.

\section{Introducción.}

Los rotíferos eurihalinos, Brachionus spp. son cultivados y utilizados como alimento vivo para larvas de peces y crustáceos en todo el mundo, la supervivencia larval en los sistemas de cultivo depende de la disponibilidad de estos, sin embargo, problemas en su producción masiva, tales como reducida reproducción o mortalidad total son causa de los mayores impactos económicos en larvicultura, por lo que el comienzo de la alimentación en estadios larvales tempranos, parece ser el mayor cuello de botella para el escalamiento industrial del cultivo de peces (Haché \& Plante, 2011; Prol-Garcia et al., 2010; Wullur et al., 2009).

Aunque estos son ideales como primer alimento exógeno, son incompletos como fuente de alimento, ya que son bajos en ácidos grasos altamente insaturados n-3 (HUFA), pues los ácidos docosahexaenoico (DHA, 22:6n-3) y eicosapentaenoico (EPA, 20:5n-3) son requeridos para el desarrollo y supervivencia en larvas de peces (Cavalin \& Weirich, 2009; Haga et al., 2006; Brown et al., 2005; Mercier et al., 2004). El enriquecimiento de rotíferos con HUFA n-3 es un proceso esencial en el campo de larvicultura marina, pudiendo estos ser alimentados y enriquecidos con microalgas seleccionadas o alimento formulado. Debido a su importancia para el crecimiento larval y desarrollo en peces, un numero de productos comerciales para enriquecimiento de rotíferos han sido desarrollados para proveer altos niveles de HUFA (Cabrera, 2008; Ando et al., 2004; Dhert et al., 2001). Sin embargo las investigaciones para comparar la eficacia de varios productos comerciales para enriquecimiento de rotíferos han sido limitadas y en el caso del rotífero nativo Brachionus sp. "Cayman", cepa Chilca, Perú, no hay información disponible, por ello el presente trabajo se realizó para evaluar el efecto de diferentes enriquecedores/suplementos alimenticios disponibles 
en el mercado local sobre la fecundidad y crecimiento de la cepa mencionada.

\section{Materiales y métodos.}

Los rotíferos utilizados en el presente estudio pertenecen al cepario del laboratorio de Alimento Vivo del Instituto del Mar del Perú. Forman parte del complejo Brachionus plicatilis, biotipo Brachionus sp."Cayman" (Romero, 2008; Dooms et al., 2007; Gomez et al., 2002). En muchos "hatcheries" se trabaja con diferentes biotipos de Brachionus sp., la designación de biotipo se utiliza para indicar una población de rotiferos con algunas diferencias genéticas entre sí y agrupados juntos en un árbol filogenético (Papakostas et al., 2009; Vasileiadou et al.,2009; Dooms et al., 2007; Gomez et al., 2002).

El biotipo Brachionus sp. "Cayman", fue aislado de muestras de agua, recolectadas entre abril y mayo del 2008, en la laguna salina "La Encantada" (12 32' 34.61' "LS y 76 43 ' 38.26" LW), distrito de Chilca, Lima. Se aclimató en el laboratorio a una salinidad de 35 ups (salinidad promedio del lugar de colecta 48 ups) y se estableció un cultivo clonal en vasos de precipitación de $500 \mathrm{~mL}$ con agua de mar filtrada (1 u) y esterilizada, a 35 ups de salinidad y $25{ }^{\circ} \mathrm{C}$ de temperatura, utilizándose la microalga Nannochloris maculata para alimentar los rotíferos aislados.

El cultivo masivo se inició con una densidad de 40 \pm 3.6 indiv. $\mathrm{mL}^{-1}$, solo en un tanque esta fue ligeramente mayor, lo cual no afecto la fecundidad, tal como se observó en los resultados. Se utilizaron tanques de acrílico de 180 litros, con agua de mar filtrada y esterilizada, a salinidad de 35 ups y temperatura constante de $27{ }^{\circ} \mathrm{C}$. Se empleó un fotoperiodo de 80:16L, ya que mayor luminosidad mejora la producción del cultivo (Yoshimatsu et al., 2008).

Se utilizaron 5 tratamientos, 4 a base de enriquecedores comerciales cuya composición se muestra en la tabla 1: Marol-E (emulsión elaborada por el SINTEF de Noruega y proporcionada por el Dr. José Rainuzzo) (T1), Protein Hufa (Salt Creek Inc., Utah, E.E.U.U) (DHA 5\%, EPA 3.5\%, proteínas 42\%) (T2), Selcon (American Marine Inc., Connecticut, E.E.U.U) ( $\sum W 3$ HUFA $200 \mathrm{mg} / \mathrm{gps}$ ) (T3) e Easy Super Selco (INVE Aquaculture, Bélgica) ( $\sum W 3$ HUFA $400 \mathrm{mg} / \mathrm{gps}$ ) (T4), así como la microalga Nannochloropsis oculata sin enriquecedor, como tratamiento control (T5). Nannochloropsis oculata $\left(6 \times 10^{7}\right.$ cel. $\left.\mathrm{mL}^{-1}\right)$ fue utilizada como alimento en todos los tratamientos con enriquecedor. Se seleccionó esta especie sobre otras algas unicelulares, por su tamaño óptimo para rotíferos (Kostopoulou \& Vadstein, 2007), su composición de lípidos (18\%), proteínas (34\%) y ácidos grasos como EPA (18\%) y ARA (2.5\%) (Banerje et al., 2011).

Los suplementos alimenticios fueron suministrados a diario por espacio de 4 días (enriquecimiento largo es más de 24 h, según Dhert et al., 2001), con frecuencias y/o intervalos según especificación del producto utilizado, en las dosificaciones siguientes: $125 \quad \mathrm{mg} . \mathrm{L}^{-1} / 350 \quad 000$ rotíferos (T1), $0.2 \mathrm{~mL} . \mathrm{L}^{-1}$ (T2), 0.1 g. $\mathrm{L}^{-1} / 1000000$ rotíferos (T3); 0.1 g. $\mathrm{L}^{-1} / 1000000$ rotíferos (T4) y una densidad de 2 x $10^{7}$ cel. $\mathrm{mL}^{-1}$ en el caso de Nannochloropsis (T5).

Diariamente se determinaron parámetros abióticos como oxígeno disuelto (OD), $\mathrm{pH}$ y amonio, así mismo para cada tratamiento se realizó el conteo de rotíferos a fin de determinar densidad poblacional (indiv. $\mathrm{mL}^{-1}$ ) y el número de huevos por hembra para determinar la fecundidad $(F)$ (huevos.indiv ${ }^{-1}$ ), la cual se calculó como (Kostopoulou \& Vadstein, 2007; Zhang et al., 2005) :

$F=$ número de huevos amicticos/ número de hembras

Se determinó la tasa de crecimiento poblacional (TC) $\left(\right.$ día $^{-1}$ ), mediante la siguiente fórmula (Rioboo et al., 2007; Suantika et al.,2002) :

$T C=(\ln N t-\ln N o) / t$

La productividad (R) en rot. $\mathrm{L}^{-1} \cdot$ día $^{-1}$ (Verginelli et al., 1994):

$R=N t-N o / t$

Donde: $N t$ es la densidad de rotíferos (indiv. $\mathrm{mL}^{-1}$ ) al tiempo $t$, No densidad inicial de rotíferos (indiv. $\mathrm{mL}^{-1}$ ) y $t$ el periodo de cultivo (días).

Análisis estadístico.

Todos los tratamientos fueron realizados por triplicado. Los datos de Productividad $(R)$ fueron transformados a escala logarítmica con el fin de aplicar el análisis estadístico. Para determinar diferencias significativas entre la $F$ y $R$, se aplicó un Análisis de Varianza de una Vía (ANOVA), seguido de la prueba de Tukey $(p<0.05)$. La normalidad de los datos y homogeneidad de varianzas, se determinaron mediante las prueba de Shapiro-Wilkins y Levene respectivamente. Para determinar la influencia del enriquecedor sobre la densidad final de rotíferos, se realizó un Análisis de Covarianza (ANCOVA). Todos los análisis se realizaron con el programa SPSS versión 19.0 .

\section{Resultados y discusión.}

\section{Parámetros de cultivo.}

Los parámetros abióticos se muestran en la tabla 1 , el amonio, oxígeno y $\mathrm{pH}$ se mantuvieron en niveles normales, la temperatura fluctuó entre 27 y $28{ }^{\circ} \mathrm{C}$. Hagiwara et al. (2007); Kotani \& Hagiwara (2003), refieren que la temperatura de cultivo influencia la fertilización de los rotíferos y la temperatura optima es diferente para las diferentes cepas de rotíferos y aun entre las mismas, pues las condiciones ambientales afectan las generaciones sucesivas.Vasileiadou et al. (2009) mencionan que las diferentes espécies de Brachionus sp. difieren en sus preferencias de temperatura, salinidad, $\mathrm{pH}$ y oxigeno disuelto. En el 
caso de la cepa estudiada en este trabajo, la temperatura mayor a $25{ }^{\circ} \mathrm{C}$ parece influenciar el crecimiento, ya que en diversas experiencias realizadas en nuestro laboratorio, se observó buen rendimiento poblacional.

En relación a los otros parámetros, el $\mathrm{pH}$ vario de 7.47 a 7.81 , OD de 5.39 a 5.85 $\mathrm{mg} / \mathrm{L}$ y los valores de NH3 ${ }^{-}$:

$\mathrm{NH} 4^{+}$fueron menores a $1 \mathrm{mg} . \mathrm{L}^{-1}$, excepto en $\mathrm{T} 2$, que fue de $1 \mathrm{mg} . \mathrm{L}^{-1}$, en este sentido, Hamre et al. (2008) mencionan que valores menores a 1 , parecen ser seguros para evitar altas mortalidades en el cultivo de rotíferos.

Tabla 1. Parámetros abióticos $(M \pm D S)$ registrados en el cultivo del rotífero Brachionus sp. "Cayman", cepa Chilca, Perú, utilizando diferentes enriquecedores.

\begin{tabular}{lccccc}
\hline Tratamientos & T1 & T2 & T3 & T4 & T5 \\
\hline temperatura $\left({ }^{\circ} \mathrm{C}\right)$ & $28.50 \pm 0.56$ & $28.10 \pm 0.50$ & $28.50 \pm 0.35$ & $28.20 \pm 0.90$ & $27.33 \pm 0.38$ \\
$\mathrm{pH}$ & $7.81 \pm 0.01$ & $7.47 \pm 0.03$ & $7.60 \pm 0.12$ & $7.73 \pm 0.02$ & $7.58 \pm 0.02$ \\
$\mathrm{OD}\left(\mathrm{mg} \cdot \mathrm{mL}^{-1}\right)$ & $5.44 \pm 0.09$ & $5.85 \pm 0.04$ & $5.79 \pm 0.18$ & $5.51 \pm 0.09$ & $5.39 \pm 0.01$ \\
$\mathrm{NH} 4^{+}-\mathrm{N}\left(\mathrm{mg}^{-1} \mathrm{~L}^{-1}\right)$ & $0.1 \pm 0.07$ & $1.05 \pm 0.06$ & $0.05 \pm 0.04$ & $0.05 \pm 0.01$ & $0.05 \pm 0.02$ \\
\hline
\end{tabular}

Fecundidad ( F).

Se observaron diferencias estadísticamente significantes (ANOVA, $p<0.05$ ) entre $F$ obtenida con los diferentes tratamientos, T2 presentó mayor $F$ a las 48 y 72 horas con $0.65 \pm 0.03$ y $0.36 \pm 0.04$ huevos.indiv. $^{-1}$ respectivamente, con valores menores a las 24 y 96 horas (tabla 2; fig. 1). Estos valores son mayores a los encontrados por Hamre et al., (2008), en un experimento realizado con una cepa del genero Brachionus especie no identificada, durante 4 días, utilizando 4 dietas a base de levadura con enriquecedores (aceite de pescado, Chlorella, algamac 2000 y Culture Selco 3000), estos autores encontraron valores de $0.32 \pm 0.01$ huevos.indiv. $^{-1}$ a las 72 horas (experimento 1) y $0.40 \pm 0.06$ huevos.indiv. ${ }^{-1}$ a las 48 horas (experimento 2).

Sin embargo, no se observaron diferencias estadísticas $(p>0.05)$ entre T2 y T5 a las 48 horas. Este resultado es similar al encontrado por Ferreira et al. (2008), en un experimento de enriquecimiento de rotíferos con la microalga Isochrysis aff. galbana (Tiso) cultivada con continua renovación del agua, quienes encontraron en los rotíferos, niveles de proteínas comparables al obtenido con dietas artificiales ricas en proteínas. Estos autores también mencionaron la importancia del contenido de las proteínas en el alimento vivo, lo que se expresa en un alto crecimiento y supervivencia de las larvas de peces, sin embargo emulsiones con ácidos grasos son requeridas para aumentar el nivel de lípidos.

Kostopoulou \& Vadstein (2007), en un estudio para determinar fecundidad y tasa de crecimiento utilizando diferentes concentraciones de Nannochloropsis oculata en el cultivo de 3 biotipos de Brachionus sp., encontraron que $B$. sp. "Cayman" presento mayor fecundidad, aún a bajas concentraciones de alimento, que $B$. plicatilis s.s. y $B$. sp. "Nevada", lo cual está asociado al corto periodo de vida del primero, el mismo que de acuerdo a lo estudiado por estos autores, invierte menos en crecimiento somático y más en crecimiento poblacional que los otros biotipos mencionados. Esto también explicaría por que en el presente trabajo la fecundidad obtenida a las 48 horas fue similar, tanto con la emulsión comercial (T2) como con la microalga (T5), cuya concentración no siempre satisface el nivel de alimento que los rotíferos requieren.

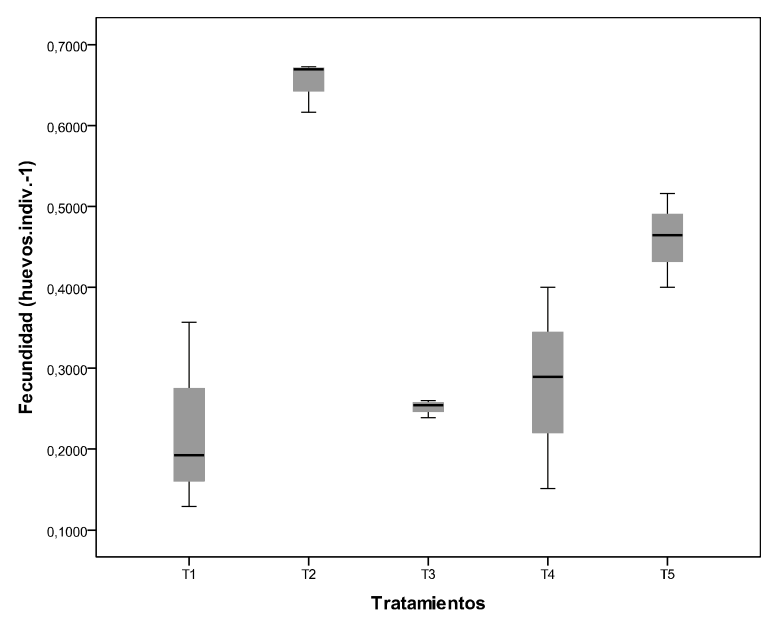

Figura 1. Fecundidad ( $\mathrm{M} \pm \mathrm{DS}$ ) (huevos.indiv ${ }^{-1}$ ), a las 48 horas de cultivo del rotífero Brachionus sp. “Cayman”, cepa Chilca, Perú, utilizando diferentes enriquecedores. 
Tasa de crecimiento (TC) $\mathrm{y}$ productividad ( $\mathrm{R}$ ).

Los resultados mostraron diferencias estadísticas $(p<0.05)$ en cuanto a $T C$ y $R$ en los diferentes tratamientos. La $T C$ fue mayor en $\mathrm{T} 2$ $\left(0.38 \pm 0.02\right.$ día $\left.^{-1}\right)$, seguido de T3 $\left(0.24 \pm 0.01\right.$ día $\left.^{-1}\right)$ (tabla 3). Según Malekzadeh Viayeh et al., (2010), la tasa de crecimiento poblacional sintetiza todos los parámetros biológicos, por que combina supervivencia, fecundidad, tiempo de desarrollo y reproducción. Los valores de $T C$ obtenidos en este trabajo con T2 y T3 fueron mayores a los encontrados por Tinh et al. (2006), en cultivo de B. plicatilis con tres tipos de alimento, Chlorella, levadura silvestre y levadura mutante mnn9, quienes encontraron valores de $0.22 \pm 002$ a $0.35 \pm 0.07 \mathrm{día}^{-1}$ con levadura, lo que se explica por la cantidad de proteínas suministradas por Protein HUFA (T2) y Nannochloropsis (en el caso de T3). Sin embargo, estos autores obtuvieron valores 0.60 a 0.69 día $^{-1}$ con Chlorella, mayor a lo encontrado en el presente trabajo con Nannochloropsis, lo que pudo deberse a que la densidad microalgal utilizada por nosotros no fue suficientemente alta para asegurar una mayor $T C$. Lo que se comprueba con los resultados de Cabrera (2008), en un experimento de cultivo con Brachionus rotundiformis alimentado con Chlorella, quien encontró valores de TC 0.37 a 1.96 día $^{-1}$, dependiendo de la densidad algal. En muchos estudios se ha investigado el efecto de diferentes dietas sobre el crecimiento (tabla 4), los cuales indican que las microalgas Chlorella y Nannochloropsis sp. son adecuadas y rotíferos alimentados con estas, pueden satisfacer los requerimientos en larvas de peces (Tinh et al., 2006). Aunque el perfil nutritivo de las microalgas, en especial de ácidos grasos se ve afectado por sus condiciones de cultivo y estos cambios pueden verse reflejados en los rotíferos (Ferreira et al., 2008).

Kostopoulou \& Vadstein (2007), en el trabajo mencionado anteriormente, también encontraron que $B$. sp. "Cayman" presentó mayor $T C$ que $B$. plicatilis s.s. y $B$. sp. "Nevada", lo cual está relacionado con su patrón reproductivo, ya que B. sp. "Cayman" tiene un tiempo de desarrollo de los huevos más corto que las 2 últimas, las que
Tabla 4. Comparación de parámetros de crecimiento entre B. sp. "Cayman" cepa chilca, Perú y especies/biotipos del complejo B. plicatilis sp.

\begin{tabular}{|c|c|c|c|c|}
\hline Especie/biotipo & $\begin{array}{c}F \\
\left(\text { huevos.indiv }{ }^{-1}\right) \\
\end{array}$ & $\begin{array}{c}C \\
\left(\text { día }^{-1}\right)\end{array}$ & $\begin{array}{c}R \\
\left(\text { rot. } L^{-1} \cdot \text { día }^{-1}\right) \\
\end{array}$ & Referencia \\
\hline B. sp. "Cayman", Perú & $0.65 \pm 0.03$ & $0.38 \pm 0.02$ & $29250 \pm 0.66$ & Presente trabajo \\
\hline B.plicatilis & & & $12130 \pm 1.89$ & Abu-Rezq \& James, 2005 \\
\hline B. plicatilis & & $0.22 \pm 0.02$ & & Tinh et al., 2006 \\
\hline B. plicatilis & & $0.33 \pm 0.09$ & & Tinh et al., 2006 \\
\hline B. plicatilis & & $0.60 \pm 0.02$ & & Tinh et al., 2006 \\
\hline B. sp. "Cayman", Italia & & $1.57 \pm 0.07$ & & Kostopoulou \& Vadstein, 2007 \\
\hline B. plicatilis & & $1.13 \pm 0.04$ & & Kostopoulou \& Vadstein, 2007 \\
\hline B. sp. "Nevada" & & $1.12 \pm 0.08$ & & Kostopoulou \& Vadstein, 2007 \\
\hline Brachionus sp. & $0.40 \pm 0.06$ & & & Hamre et al., 2008 \\
\hline B. rotundiformis & & 0.37 a 1.00 & & Cabrera, 2008 \\
\hline
\end{tabular}


proveen un mejor rendimiento poblacional, por su composición de HUFA's n-3 y proteinas, cuyos niveles son significantemente más altos en rotíferos cultivados con estos que con mixtura de algas y levadura (Dhert et al., 2001).

El ANCOVA demostró que el efecto del suplemento sobre la densidad final fue significativa ( $p$ $=0.0001$ ), así mismo que el $99 \%$ de las variaciones de la densidad final obtenida en el cultivo, fueron explicables por el enriquecedor, encontrándose que T2 fue el más eficiente, con el cual se obtuvo una densidad final promedio de $149 \pm 0.58$ indiv. $\mathrm{mL}^{-1}$ (fig. $3)$.

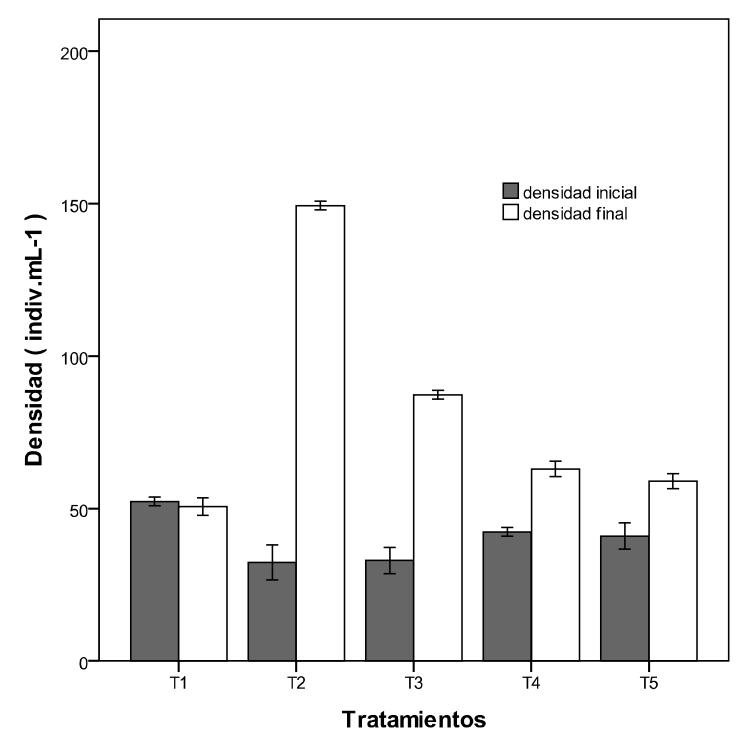

Figura 3. Densidad inicial y final $(M \pm D S)$ (indiv. $\mathrm{mL}^{-1}$ ) obtenidas en el cultivo del rotífero Brachionus sp. "Cayman", cepa Chilca, Perú, utilizando diferentes enriquecedores.

El costo beneficio de la producción de rotíferos recae en el uso de una fuente de alimento barata, por ello se explica que en muchos casos la levadura de panificación sea usada como dieta, sin embargo esta carece de los ácidos grasos requeridos. En este sentido cuando hay disponibilidad de microalgas de buena calidad estas pueden usarse como alimento vivo por su contenido de ácidos grasos, como es el caso de Chlorella, Nannochloropsis occulata e Isochrysis galbana, aunque hay que tener en cuenta que los costos de producción son más elevados cuando se utilizan microalgas. Para asegurar el nivel nutricional de los rotíferos, se utilizan emulsiones lipidicas, enriqueciéndolos unas horas antes de suministrarlos como alimento a las larvas, a esto se conoce como enriquecimiento corto (8 horas a menos) (Dhert et al., 2001). La ventaja de esta técnica es que es rápida y flexible, pero con frecuencia produce baja calidad en cuanto a higiene, por el alto contenido de grasa acumulada durante el proceso de enriquecimiento
(Dhert et al., 1990; Stottrup \& Attramadal, 1992) (en Dhert et al., 2001), ya que los rotíferos son concentrados a altas densidades y pese al lavado restos de la emulsión se transfiere a los tanques de larvas. En este sentido Haché y Plante (2011) demostraron que después del proceso de enriquecimiento con emulsiones comerciales (durante 24 horas), se incrementó el número de colonias de bacterias, en un estudio realizado con Brachionus plicatilis, estos mismos autores mencionan que el alimento vivo de por sí, representa un significante vector de contaminación bacteriana. Adicional a esto Dhert et al., (2001) mencionan que la retención de nutrientes en el tracto digestivo de los rotíferos es corto, presentándose inconvenientes si estos no son ingeridos inmediatamente y que la ventaja del enriquecimiento largo (más de $24 \mathrm{~h}$ ) es que los rotíferos alimentados siguiendo esta estrategia de alimentación son nutricionalmente más estables y pierden sus reservas más lentamente. En este sentido desde que se comenzó a utilizar dietas complementarias, productos como Protein Selco (PS) y DHA Culture Selco (DHA- CS) han sido lanzados al mercado, a fin de incorporar niveles más altos de proteínas y DHA, ácido graso altamente insaturado, esencial para el crecimiento larvario en peces (King et al., 2002).

\section{Conclusiones.}

En el presente trabajo el suplemento Protein Hufa proporciono mejores resultados, en cuanto a fecundidad y productividad, debido a su alto nivel de proteínas (42\%), seguido de Easy Super Selco, cuyo nivel de lípidos es más alto (67\%), por lo que concluimos que es posible obtener un buen rendimiento poblacional en el cultivo del rotífero nativo Brachionus sp. "Cayman", cepa Chilca, Perú, utilizando una fuente rica en proteínas y ácidos grasos, siendo recomendable el enriquecimiento largo, con una dieta que asegure el crecimiento poblacional, así como la estabilidad nutricional de los rotíferos, esto se puede complementar con el enriquecimiento corto. Sin embargo ya que diferentes alimentos/suplementos comerciales han sido lanzados al mercado, es necesario realizar más investigaciones orientadas a mejorar las estrategias de enriquecimiento de la cepa estudiada en este trabajo, haciendo un análisis del contenido de ácidos grasos.

\section{Agradecimientos.}

El presente trabajo se realizó con financiamiento del Instituto del Mar del Perú (IMARPE), en el marco del Proyecto "Cultivo de Organismos Marinos de importancia Económica". Se agradece al Ing. Marco de la Cruz por su apoyo durante la ejecución del trabajo. 


\section{Literatura citada.}

Ando Y., Kobayashi S., Sugimoto T. \& Takamaru N. 2004. Positional distribution of $n-3$ higly unsatured fatty acids in triagly-sn-glycerol (TAG) of rotifers (Brachionus plicatilis) enriched with fish and seal oils TAG. Aquaculture. 2004: 275-288.

Abu-Rezq T. \& James C. 2005. Application of probiotics in rotifer production systems for marine fish hatcheries. Marine finfish Aquaculture Network. July - September 2005.

Banerjee S, Hew W E, Khatoon H, Shariff M \& and Yusoff F. 2011. Growth and proximate composition of tropical marine Chaetoceros calcitrans and Nannochloropsis oculata cultured outdoors and under laboratory conditions. Afr. J. Biotechnol Vol. 10(8) : 1375-1383.

Brown M.R., Dunstan G. A., Nichols P.D., Battglene S.C., Morhead D.T. \& Overweter A.L. 2005. Effects of $\alpha-$ tocoferol supplementation of rotifer on the growth of striped trumpeter Latris lineata larvae. Aquaculture. 246: 367- 378.

Cabrera M.I. 2008. Tasa de crecimiento ploblacional del rotífero Brachionus rotundiformis (Rotifera: Brachionidae) en un quimiostato de dos cámaras. Rev. Biol. Trop. 56: 1149-1157.

Cavalin F.G.\& Weirich C.R. 2009. Larval performance of aquacultured Florida pompano (Trachinotus carolinus) fedrotifers (Brachionus plicatilis) enriched with selected commercial diets. Aquaculture $292: 67-73$

Dhert P, Rombaut G, Suantika G \& Sorgeloos P. 2001 Advancement of rotifer culture and manipulation techniques in Europe. Aquaculture 200 : 129-146.

Dooms S., Papakostas S., Hoffman S., Delbare D., Dierckens K., Triantafyllidis A., de Wolf T., Vadstein O., Abatzopoulos T. J., Sorgeloos P. \& Bossier P. 2007. Denaturing Gradient Gel Electrophoresis (DGGE) as a tool for the characterisation of Brachionus sp. Strains. Aquaculture. 262: 29-40.

Ferreira M., Maseda A., Fabregas J. \& Otero A. 2008 Enriching rotifers with "premium" microalgae. Isochrysis aff. galbana clone T-ISO. Aquaculture 279: 126-130.

Gomez A., Serra M., Carvalho G. \& Lunt D.H. 2002. Speciation in ancient cryptic species complexes: evidence from themolecular phylogeny of Brachionus plicatilis (rotifera). Evolution, 56(7), 2002, pp. 14311444.

Haché R. \& Plante S. 2011. The relationship between enrichment, fatty acid profiles and bacterial load in cultured rotifers (Brachionus plicatilis L-strain) and Artemia (Artemia salina strain Franciscana). Aquaculture 311 (2011) 201-208.

Haga Y., Tarui F., Ohta K., Shima Y. \& Takeuchi T. 2006. Effect of light irradiation on dynamics of vitamin A compound in rotifer and Artemia. Fisheries Science. 72: $1020-1026$

Hagiwara A., Suga K., Akazawa A., Kotani T. \& Sakakura Y. 2007. Development of rotifer strains with useful traits for rearing fish larvae. Aquaculture 268: 44-52.

Hamre A., Srivastava A., RØnnestad I., Mangor-Jensen A. \& Stoss J. 2008. Several micronutrients in the rotifer Brachionus sp. may not fulfil the nutritional requirements of marine fish larvae. Aquaculture Nutrition. 14: 51-60.
King J., Xiaoming L. \& Rusch K. 2002. Nutritional properties of the marine rotifer Brachionus plicatilis fed the fresh water microalgae Selenastrum capricornutum. World Aquaculture Society. 33: 478-488.

Kostopoulou V. \& Vadstein O. 2007. Growth performance of the rotifers Brachionus plicatilis, B. 'Nevada' and $B$. 'Cayman' under different food concentrations. Aquaculture273 : 449 - 458.

Kotani T. \& Hagiwara A. 2003. Fertilization between rotifer Brachionus plicatilis strains at different temperatures. Fisheries Science 69:1078-1080.

Malekzadeh Viayeh R., Mohammadi H. \& Shafiei A. B. 2010. Population growth of six Iranian Brachionus rotifer strainsin response to salinity and food type. Internat. Rev. Hydrobiol. 95, $6: 461-470$.

Mercier L., Audet C., de la Noue J., Parent B., Parrish C.\& Ross N. W. 2004. First feeding of winter flounder (Pseudopleuronectes americanus) larvae : use of Brachionus plicatilis acclimated at low temperature as live prey. Aquaculture $229: 361-376$.

Papakostas S., Triantafyllidis A., Kappas I. \& Abatzopoulos T.J. 2009. Clonal composition of Brachionus plicatilis s.s and B. sp. 'Austria' Hatchery strains based on microsatellite data. Aquaculture. 296: 15-20

Prol-Garcia M.J., Planas M. \& Pintado J. 2010. Different colonization and residence time of Listonella anguilarum and Vibrio splendidus in the rotifer Brachionus plicatilis determined by real-real time PCR and DGGE. Aquaculture 302 (2010) 26-35.

Rioboo C., Prado R., Herrero C., Cid A. 2007. Population growth study of the rotifer Brachionussp. fed with triazine-exposed microalgae. Aquatic Toxicology 83 : 247-253.

Romero L. 2008. Caracterización morfométrica y aspectos filogenéticos de cepas de rotíferos del grupo Brachionus plicatilis (Rotifera: Brachionidae) utilizados en la acuicultura peruana. TESIS para optar el título profesional de Biólogo. Facultad de Ciencias Biologicas, UNMSM. 76pp.

Suantika G., Dhert P., Rombaut G., Vandenbergue J., De Wolf T. \& Sorgeloos P. 2002. The use of ozone in a high density recirculation system for rotifers. Aquaculture. 201: 35-49.

Tinh N, Phuoc N, Dierckens K, Sorgeloos P \& Bossier P. 2006. Gnotobiotically grown rotifer Brachionus plicatilis sensu strictu as atool for evaluation of microbial functions and nutritional value ofdifferent food types.Aquaculture 253:421- 432.

Vasileiadou K., Papakostas S., Triantafyllidis A., Kappas I. \& Abatzopoulos T. 2009. A multiplex PCR method for rapid identification of Brachionus rotifers. Mar biotechnol (2009) 11:53-61.

Verginelli R., Marín N. \& Lodeiros C.1994. Cultivo masivo del rotíferoBrachionus plicatilis con tres dietas de microalgas. Rev. Lat. Acui. Lima-Perú, $N^{\circ} 43$ : 68-71 pp.

Wullur S., Sakakura Y. \& Hagiwara A. 2009. The minute monogonont rotifer Proales similis de Beauchamp: Culture and feeding to small mouth marine fish larvae. Aquaculture 293 (2009) 62-67.

Yoshimatsu T., Higuchi T., Hamasaki Y. \& Tanaka K. 2008. Preliminary Trials on the Effects of Lighting for the Populations Growth of the Rotifer, Brachionus plicatilis. JARQ. 42: 131-136. 
Zhang D.M., Yoshimatsu T. \& Furuse M. 2005. Effects of L-carnitine enrichment on the population growth, egg ratio and body size of the marine rotifer, Brachionus rotundiformis. Aquaculture. 248: 51-57.

\footnotetext{
${ }^{1}$ Unidad de Investigaciones en Acuicultura, Instituto del Mar del Perú, Apartado 22 , Chucuito, Callao, rcisneros@imarpe.gob.pe.
} 